\title{
A DFT reinvestigation of chemo- and stereoselectivity epoxidation from $\alpha$ - and $\gamma$-trans himachalene with meta Chloroperoxybenzoic acid
}

\author{
Abdelhak Ouled Aitouna ${ }^{1, *}$, Lahoucine Bahsis ${ }^{2}$, Hicham Ben El Ayouchia ${ }^{2, *}$, Ahmed Benharref ${ }^{1}$, Redouan \\ Hammal ${ }^{1}$ and Ahmed Chekroun ${ }^{1}$ \\ ${ }^{1}$ Laboratory of Chemistry and Biomolecular, Natural Substances and Reactivity, Faculty of Sciences Semlalia, \\ Cadi Ayyad University, B.P. 2390, 40000 Marrakech, Morocco \\ ${ }^{2}$ Laboratory of Analytical and Molecular Chemistry, LCAM, Polydisciplinary Faculty, Cadi Ayyad University, \\ B.P. 46030, Safi, Morocco
}

\begin{abstract}
In this work the epoxidation reaction of the $\alpha$ - and $y$-trans himachalene in the presence of meta chloroperoxybenzoic acid (m-CPBA) has been studied within the Density Functional Theory (DFT) method at the B3LYP/6-311G $(\mathrm{d}, \mathrm{p})$ level in dichloromethane as a solvent, in order to shed light on the chemo- and stereoselectivity in the course of the reaction. Analysis of the Conceptual Density Functional Theory (CDFT) reactivity indices indicate that the $\mathbf{m}$-CPBA will behave as electrophilic while $\alpha$ - and $\gamma$-trans himachalene will behave as a nucleophile and the attacks observed experimentally are correctly predicted by the electrophilic $\mathrm{P}_{\mathrm{k}}{ }^{+}$ and nucleophilic $\mathrm{P}_{\mathrm{k}}-$ Parr functions. The two reactive paths associated with chemo and stereoselectivity approach modes of $\mathbf{m}$-CPBA on $\mathrm{C}=\mathrm{C}$ reactive sites in $\alpha$ and $\gamma$-trans himachalene have been analyzed. They showed that m-CPBA reacted as electrophile whereas $\alpha$ - and $\gamma$ - trans himachalene as a nucleophile. The Monoepoxidation of $\alpha$ - and $\gamma$ - trans himachalene leads to the formation of two stereoisomers, on the most substituted double bond " $\mathrm{C}=\mathrm{C}$ », one of the two is a majority. The diepoxidation reaction of $\alpha$ - and $\gamma$ - trans himachalene in the presence of $\mathbf{m}$-CPBA gives two more stereoisomers in the other carbon-carbon double bond.
\end{abstract}

Keywords: Epoxidation m-CPBA; $\alpha$-trans himachalene; $\mathrm{\gamma}$-trans himachalene; DFT; Dichloromethane.

\section{Introduction}

During the past decades, essential oils are ubiquitous in our daily lives: in hygiene products, room fragrances, in aromatic oils for wellness massages, marketed as complexes to purify our air polluted and get rid of mites or other insects, or recommended in the kitchen to customize some dishes. The use of aromatherapy, today very "trendy", dates back to very old times ${ }^{1}$. The essential oil of the Atlas Cedar is primarily constituted $(75 \%)$ of three sesquiterpene bicyclic hydrocarbons: $\alpha$-cis-himachalene, $\beta$-himachalene, and $\gamma$-cis-himachalene which is used in the perfume industry ${ }^{2}$. Each one of them differs from the others only by the seven-membered ring double bond positions ${ }^{3}$. trans himachalenes are synthesized from the three sesquiterpene hydrocarbons (cis himachalenes) ${ }^{4}$. Benharef et al. experimentally reported the synthesis of epoxides from $\alpha$-trans-himachalene and $\gamma$-trans-himachalene by using epoxidation agent well known in organic chemistry such as m-CPBA ${ }^{4,5}$. The epoxide obtained is an important compound which used as an intermediate in medical chemistry ${ }^{6,7}$, as well as polyvalent components in the synthesis of natural products ${ }^{8}$, and biologically active compounds ${ }^{9,10}$. The epoxidation reaction of $\alpha$ and $y$-trans himachalene in the presence of $\mathbf{m}$-CPBA take place at the terminal oxygen atom of the peroxy acid. Thus, A single equivalent of m-CPBA led to attacking the double bond $\mathrm{C} 2=\mathrm{C} 3$ and $\mathrm{C} 11=\mathrm{C} 13$ of (1-2) respectively producing preferentially the product "out" (Scheme 1). The reaction with a second equivalent led to an attack on the double liaison that remains. Very few theoretical studies devoted to epoxidation reaction are found in the literature ${ }^{2}$. By performing theoretical calculations concerning the behavior of (1-2) in the presence of m-CPBA, we were able to predict the selectivity using reactivity index and activation energies of transition states (TS). The two transition states, namely, TS out $_{\text {and }} \mathbf{T} S_{\text {ins }}$ corresponding the two attack modes are located at the B3LYP/6-311G(d,p) level of the theory (Scheme 1). Activation and reaction energies are calculated for the two reaction channels to put in evidence the kinetic and thermodynamic controls of these epoxidations. 


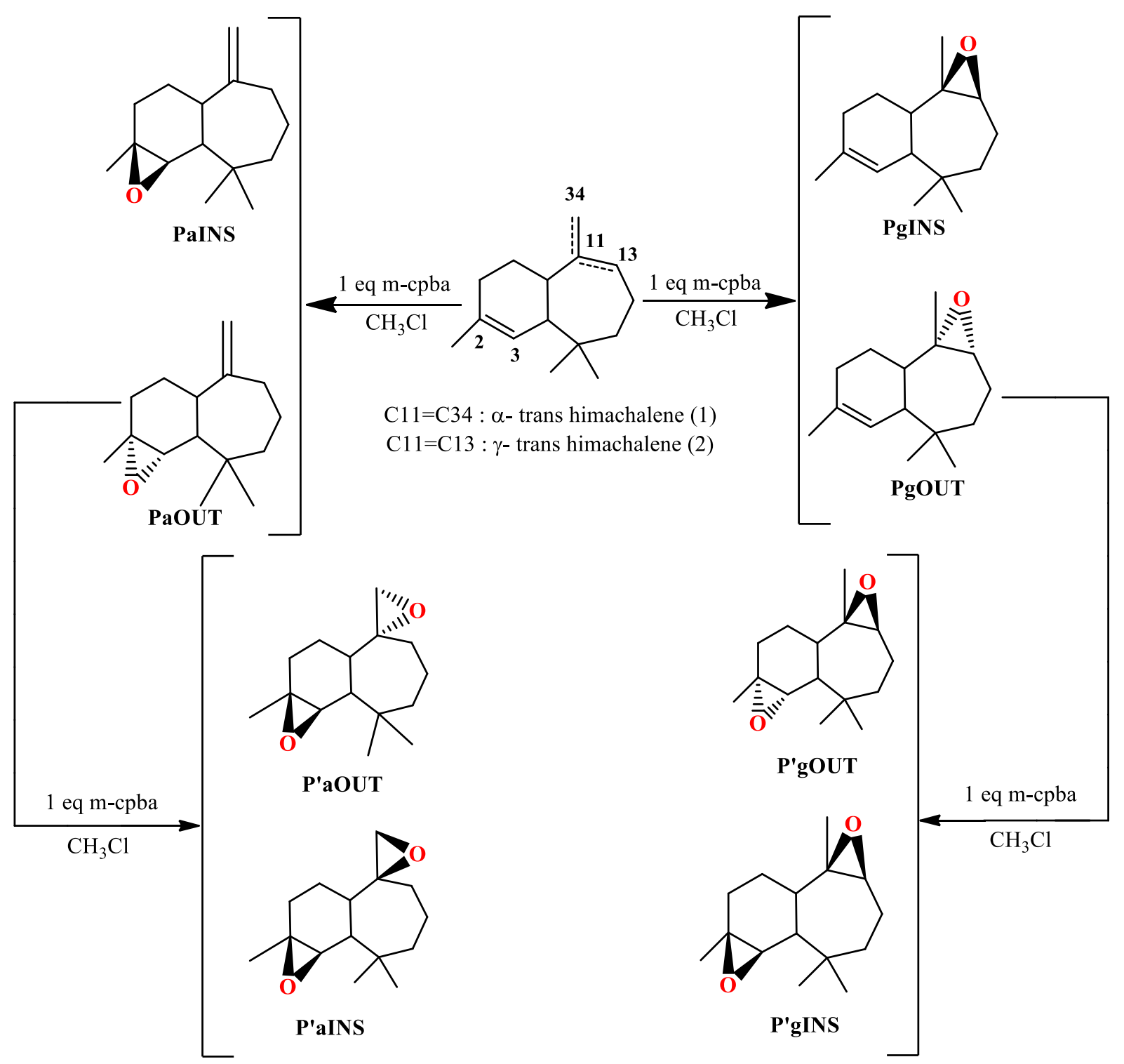

Scheme 1. Pathways of epoxidation reaction of $\alpha$ - and $\gamma$-trans himachalene in presence of $\mathbf{m}$-CPBA.

\section{Computational details}

All calculations reported in this study have been performed using DFT method at B3LYP/ $6-311 G(d, p)$ level of theory using the Gaussian 09 series of programs ${ }^{11-14}$. The optimizations at this level of theory were performed using the Berny analytical gradient optimization method ${ }^{15}$. Solvent effect of dichloromethane (DCM) was considered using the polarizable continuum model (PCM) developed by Tomasi's group in the framework of the self-consistent reaction field (SCRF) ${ }^{16,17}$. The transition states (TSs) have been located at B3LYP/6-311G(d, p) level of theory. The stationary points were characterized by calculations of frequency (performed at the same level of theory) to verify that the TSs had one and only imaginary frequency. Intrinsic reaction coordinate (IRC) calculations were also conducted to ensure the TSs indeed connect two relevant minima on the potential energy surface.
The global electrophilicity index $\omega$ is given by the following expression ${ }^{18}, \omega=\left(\mu^{2}\right) / 2 \eta$ in terms of the electronic chemical potential $\mu$ and the chemical hardness $\eta$. Both quantities may be approached in terms of the one-electron energies of the frontier molecular orbital HOMO and LUMO, as $\varepsilon_{\text {HOMO }}$ and $\varepsilon_{\mathrm{LUMO}}, \mu \approx\left(\varepsilon_{\mathrm{HOMO}}+\varepsilon_{\mathrm{LUMO}}\right) / 2$ and $\eta \approx \varepsilon_{\mathrm{LUMO}}-\varepsilon_{\mathrm{HOMO}}$, respectively ${ }^{19,20}$. The nucleophilicity index $N^{21,22}$, based on the HOMO energies obtained within the Kohn-Sham scheme ${ }^{23}$, and defined as $N=\varepsilon_{\mathrm{HOMO}(\mathrm{Nu})}-$ $\varepsilon_{\text {HOMO(TCE). The nucleophilicity is referred to }}$ tetracyanoethylene (TCE). The electrophilic $\mathrm{P}_{\mathrm{k}}{ }^{+}$and nucleophilic $\mathrm{P}_{\mathrm{k}}$ - Parr functions ${ }^{24-32}$, which allow for the characterization of the electrophilic and nucleophilic centers of a molecule, were obtained through the analysis of the Mulliken atomic spin density and can be redefined as follows: $\omega \mathrm{k}=\omega \times P_{k}{ }^{+}$ and $N_{k}=N \times P_{k}^{-}$. Although, we note that the anionic and cationic systems, required in the calculations of the reactivity indexes were kept at the same optimized geometry. 


\section{Results and Discussion}

\subsection{Monoepoxidation reaction}

3.1.1. Chemical descriptors of $\alpha$, and $\mathrm{z}$-trans himachalene and $\mathrm{m}$-CPBA

The conceptual DFT (CDFT) is a first method to appreciate the reactivity in extreme reactions 33,34 .
Therefore, the global CDFT indices, namely, the chemical potential, $\mu$, chemical hardness, $\eta$, electrophilicity, $\omega$, and nucleophilicity, $N$, of m-CPBA, $\alpha$-trans himachalene $\mathbf{1}$ and $\gamma$-trans himachalene $\mathbf{2}$ are given in Table 1.

Table 1. Electronic chemical potential, global electrophilicity and global nucleophilicity in eV.

\begin{tabular}{|l|l|l|l|}
\hline Compounds & $\boldsymbol{\mu}(\mathbf{e V})$ & $\boldsymbol{\omega}(\mathbf{e V})$ & $\boldsymbol{N}(\mathbf{e V})$ \\
\hline $\boldsymbol{\alpha}$-trans himachalene (1) & -3.03 & 0.69 & 2.68 \\
\hline J-trans himachalene (2) & -2.84 & 0.60 & 2.85 \\
\hline m-CPBA & -4.76 & 2.16 & 1.65 \\
\hline
\end{tabular}

According to the values quoted in the Table 1 , we can notice that the electronic chemical potential of $\alpha$-trans himachalene (1) $(-3.03 \mathrm{eV})$ and $\gamma$-trans himachalene (2) $(-2.84 \mathrm{eV})$ are higher than the electronic chemical potential of $\mathbf{m}-\mathbf{C P B A}(-4.76 \mathrm{eV})$, which means that the electron transfer takes the place of (1) and (2) to m-CPBA. The global electrophilicity of the $\mathbf{m - C P B A}(2.16 \mathrm{eV})$ is greater than that of (1) $(0.69 \mathrm{eV})$ and (2) $(0.60 \mathrm{eV})$.

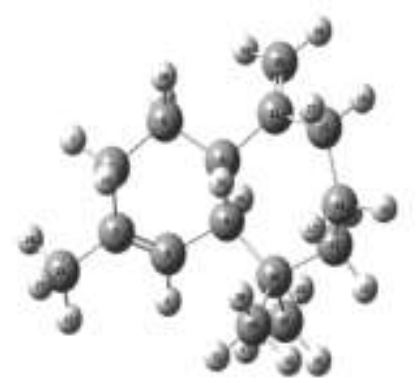

(a)

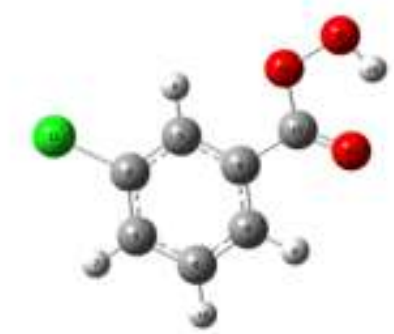

Therefore, in this cycloaddition reaction, the $\mathbf{m}$ CPBA will behave as electrophilic while the (1) and (2) will behave as the nucleophile that is confirmed by the nucleophilicity values. Figure 1 shows the optimized structures of $\alpha$-trans himachalene (1), mCPBA and $\mathrm{y}$-trans himachalene (2). The results obtained theoretically are in agreement with the experimental results ${ }^{4,5}$.

(b)

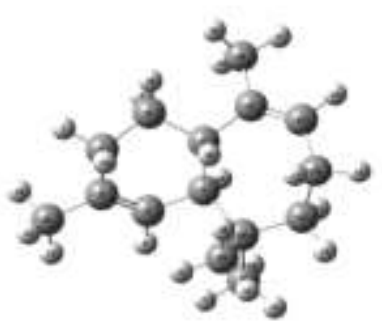

(c)

Figure 1. Optimized geometries of $\alpha$-trans himachalene (1) (a), m-CPBA (b) and $\gamma$-trans himachalene (2) (c).

\subsubsection{Comparative analysis of local indices for} reagents

The values of local electrophilicity $\omega_{\mathrm{k}}\left(\omega_{\mathrm{k}}=\omega_{\mathrm{k}} \cdot \mathrm{P}_{\mathrm{k}}{ }^{+}\right)$ for m-CPBA and the local nucleophilicity $N_{k}$
$\left(N_{k}=N . P_{k}^{-}\right)$for atoms of $\alpha$ and $\gamma$ trans -himachalene calculated with the function Parr are reported in Table 2 .

Table 2. Electrophilic and Nucleophilic Parr Functions, local electrophilicity and nucleophilicity.

\begin{tabular}{|c|c|c|c|c|c|}
\hline & Atoms & $\mathbf{P}_{\mathbf{k}}^{+}$ & $\mathbf{P}_{\mathbf{k}}^{-}$ & $\omega_{k}$ & $N_{k}$ \\
\hline \multirow{4}{*}{$\begin{array}{l}\alpha \text {-trans himachalene } \\
\text { (1) }\end{array}$} & $\mathrm{C} 2$ & - & 0.28 & - & 0.75 \\
\hline & $\mathrm{C} 3$ & - & 0.36 & - & 0.96 \\
\hline & C11 & - & 0.08 & - & 0.21 \\
\hline & C34 & - & 0.15 & - & 0.41 \\
\hline \multirow[t]{3}{*}{ m-CPBA } & O13 & 0.00 & - & 0.00 & - \\
\hline & O14 & 0.03 & - & 0.07 & - \\
\hline & O15 & 0.12 & - & 0.27 & - \\
\hline \multirow{4}{*}{$\begin{array}{l}\text { y-trans himachalene } \\
\text { (2) }\end{array}$} & $\mathrm{C} 2$ & - & 0.18 & - & 0.29 \\
\hline & $\mathrm{C} 3$ & - & 0.18 & - & 0.30 \\
\hline & C11 & - & 0.19 & - & 0.32 \\
\hline & C13 & - & 0.29 & - & 0.47 \\
\hline
\end{tabular}


These results show that the most favored interaction takes place between the $\mathrm{O} 15$ oxygen atom of m-CPBA (with the highest value of $\omega_{\mathrm{k}}$ ) and the $\mathrm{C} 2$ and $\mathrm{C} 3$ atoms of $\alpha$-trans himachalene (C11 and $\mathrm{C} 13$ of y-trans himachalene) (with the highest $N_{k}$ value). Consequently, the attacks observed experimentally are correctly predicted by Parr function. In the end, point out that the attack of the $\mathrm{C} 2=\mathrm{C} 3 \alpha$-trans himachalene double bond (C11 and $\mathrm{C} 13$ of $\mathrm{y}$-trans himachalene) is preferable, hence the need to talk about stereoselectivity.

\subsubsection{Stereoselectivity of reaction between (1-2) and $\mathrm{m}$-CPBA}

The studied epoxidation reactions can take place via two possible reactive channels corresponding two transition states; namely, TSout and TSins have been located and characterized by calculations of frequency. The geometries of the two TSs is given in Scheme1 together with the two new forming bonds lengths. Moreover, the relative energies of all intermediates are calculated and the results are presented in Table 3.

For $\alpha$-trans himachalene (1), TSout is located at $7.84 \mathrm{kcal} . \mathrm{mol}^{-1}$ and is located at $6.46 \mathrm{kcal}^{\mathrm{mol}}{ }^{-1}$ for y-trans himachalene (2) which are low to those obtained for inside reactions (TSins of $(\mathbf{1})$ is located at $10.35 \mathrm{kcal} . \mathrm{mol}^{-1}$ and for (2), it is located at 9.78 kcal.mol ${ }^{-1}$ ). These results confirm that the outside pathway reaction is kinetically more favorable. Moreover, the small difference in energy between TSout and TSins for $(\mathbf{1}),\left(\Delta \Delta \mathrm{E} \#=2.51 \mathrm{kcal}^{\mathrm{m}} \mathrm{mol}^{-1}\right)$ and for (2) $\left(\Delta \Delta \mathrm{E} \#=3.32 \mathrm{kcal} . \mathrm{mol}^{-1}\right)$, indicating a weak stereoselectivity.

The thermodynamic profiles of the reaction paths between $\alpha$-trans himachalene (1), $\gamma$-trans himachalene (2) and $\mathbf{m - C P B A}$, with the relative enthalpies $(\Delta H)$ and Gibbs free energies $(\Delta G)$, are summarized in Table 3. The inclusion of thermal corrections to the electronic energies does not substantially modify the relative enthalpies; while the activation enthalpies slightly increase by ca. 0.1 kcal.mol ${ }^{-1}$. However, the inclusion of entropies to enthalpies sharply increases the activation Gibbs free energies by between 10 and $12 \mathrm{kcal}^{\mathrm{mol}}{ }^{-1}$, which indicate that the most favorable reaction path corresponds to the formation of the product via TSout.

Table 3. Energy $(\Delta \mathrm{E})$, Enthalpy $(\Delta \mathrm{H})$ and Gibbs free energy $(\Delta \mathrm{G})$ profiles, in $\mathrm{kcal} / \mathrm{mol}$, for the monoepoxidation reaction paths between (1-2) and $\mathbf{m}-\mathbf{C P B A}$.

\begin{tabular}{|l|l|l|l|}
\hline Compounds & $\mathbf{\Delta E}$ & $\boldsymbol{\Delta H}$ & $\boldsymbol{\Delta}$ \\
\hline $\boldsymbol{\alpha}$-trans himachalene (1) & - & - & - \\
\hline J-trans himachalene (2) & - & - & - \\
\hline m-CPBA & - & - & - \\
\hline TSout(1) & 7.84 & 7.90 & 18.69 \\
\hline TSins(1) & 10.35 & 10.41 & 22.40 \\
\hline TSout(2) & 6.46 & 6.33 & 16.94 \\
\hline TSins(2) & 9.78 & 9.72 & 20.64 \\
\hline PaOUT & -51.58 & -122.67 & -93.93 \\
\hline PaINS & -50.26 & -121.23 & -92.61 \\
\hline PyOUT & -54.34 & -125.50 & -96.88 \\
\hline PyINS & -51.07 & -122.11 & -93.62 \\
\hline
\end{tabular}

The studied epoxidation reactions can take place via two possible reactive channels corresponding two transition states, namely, TSout and TSins have been located and characterized by calculations of frequency. The geometries of the two TSs is given in Scheme 1 together with the two new forming bonds lengths. Moreover, the structure of TSout and TSins are given in Table 4. The lengths of $\mathrm{O}-\mathrm{C}$ forming bonds at the regioisomeric for TSout and TSins, respectively are: For $(\mathbf{1})$ : $(\mathrm{C} 2-\mathrm{O} 15)=2.275 \AA$ and $(\mathrm{C} 3-$ $\mathrm{O} 15)=2.244 \AA ; \quad(\mathrm{C} 2-\mathrm{O} 15)=2.187 \AA \quad$ and $\quad(\mathrm{C} 3-$ $\mathrm{O} 15)=2.335 \AA$. For $(2)$ : $(\mathrm{C} 11-\mathrm{O} 15)=2.409 \AA$ and $(\mathrm{C} 13-\mathrm{O} 15)=2.209 \AA ;(\mathrm{C} 11-\mathrm{O} 15)=2.334 \AA$ and $(\mathrm{C} 13$ $\mathrm{O} 15)=2.238 \AA$.
Recently, many studies have shown a strong relationship between the polar character and the feasibility of organic reactions, and the broader global electron density transfer (GEDT) at the TS is, the more polar and thus the faster is the reaction ${ }^{35}$. The polar nature of the epoxidation reactions has been analyzed by calculation of the GEDT at the TSs, and the corresponding GEDT values are reported in (Table 4). The natural population analysis shows that the electron-density flow takes place from (1) or (2) to m-CPBA In monoepoxidation reactions, the values of the GEDT are $0.348 \mathrm{e}$ at TSains, $0.340 \mathrm{e}$ at TSaout, $0.311 \mathrm{e}$ at TSyins, and $0.344 \mathrm{e}$ at TSyout. 
Table 4. Optimized geometries of the stationary points involved in the monoepoxidation reaction of the $\alpha$ - and $\gamma$-trans himachalene and $\mathbf{m - C P B A}$. The lengths are given in angstroms, while the angles are given in degrees

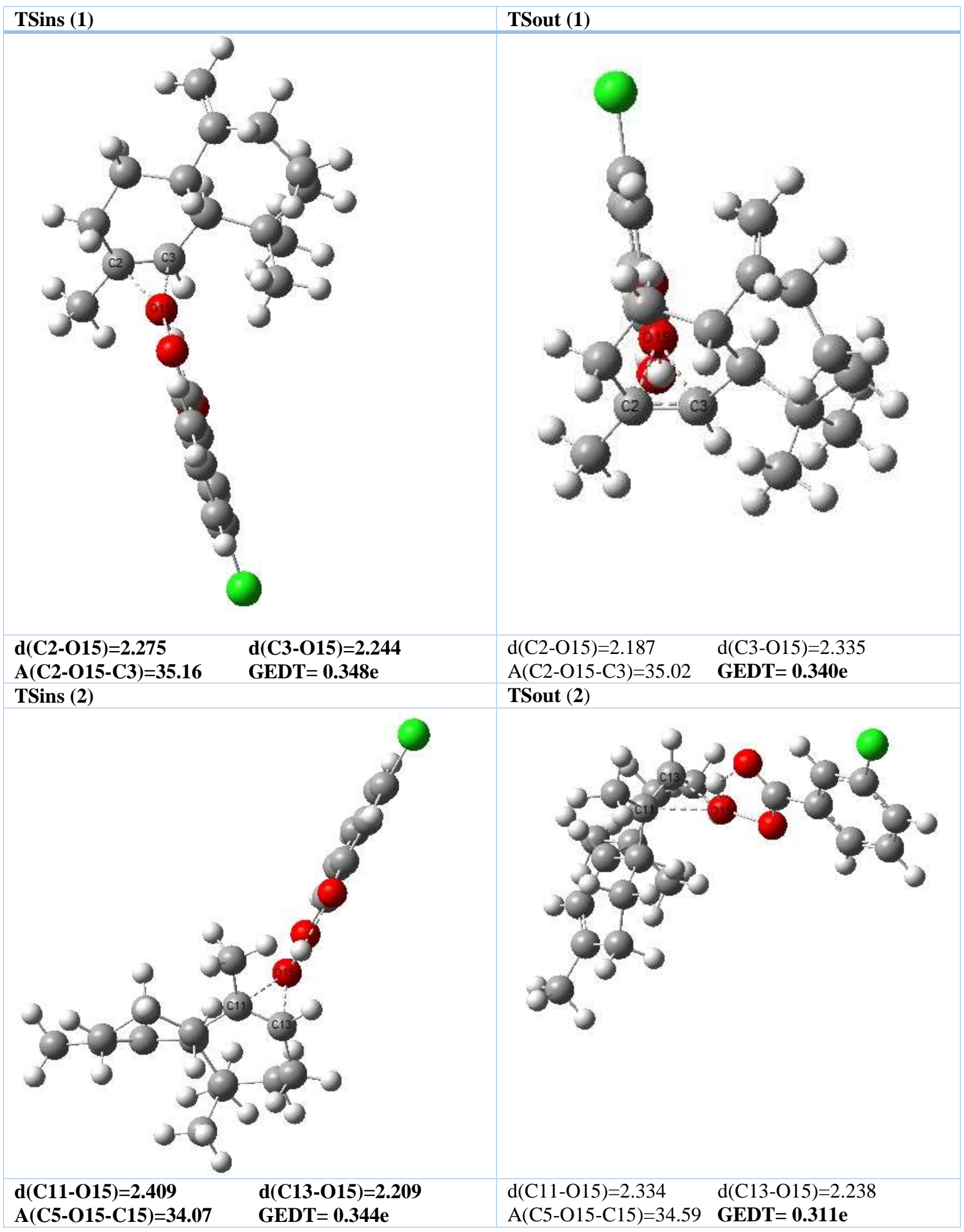

\subsection{Diepoxydation reaction}

3.2.1. Chemical descriptors of the PaOUT, PyOUT and m-CPBA

The electrophilicity index of m-CPBA $(2.16 \mathrm{eV})$, are higher than that of the reagents $(3)$ and $(4)(0.74 \mathrm{eV}$ and $0.63 \mathrm{eV})$. Therefore, in this epoxidation, the $\mathbf{m}$ -

CPBA behaves as an electrophile while (3) and (4) will behave like nucleophiles (Table 5). The chemical potential of (3) and (4) $(-3.21 \mathrm{eV}$ and $2.96 \mathrm{eV}$ ) is higher than that of $\mathbf{m}-\mathbf{C P B A}-4.76 \mathrm{eV})$, which implies that the electron transfer takes place from PaOUT, PyOUT to the m-CPBA. 
Table 5. Electronic chemical potential, global electrophilicity and global nucleophilicity in eV.

\begin{tabular}{|l|l|l|l|}
\hline Compounds & $\boldsymbol{\mu}(\mathbf{e V})$ & $\boldsymbol{\omega}(\mathbf{e V})$ & $\boldsymbol{N}(\mathbf{e V})$ \\
\hline PaOUT(3) & -3.21 & 0.74 & 2.35 \\
\hline PyOUT(4) & -2.96 & 0.63 & 2.63 \\
\hline m-CPBA & -4.76 & 2.16 & 1.65 \\
\hline
\end{tabular}

According to our calculations, we found the same value of the transitions energies TSout and TSins for the reagent (3) (TSout is lower than TSins) (Table 6). For the reagent (4), the formation of P'yOUT is more energetically favorable, which are in good agreements with the experimental results ${ }^{4,5}$.

The thermodynamic profiles of the epoxidation reaction paths between PaOUT, PyOUT, and m-CPBA are summarized in Table 6. The results demonstrated that the inclusion of thermal corrections to the electronic energies does not substantially modify the relative enthalpies; while the activation enthalpies slightly increase by ca. 0.2 kcal.mol ${ }^{-1}$. However, the inclusion of entropies to enthalpies strongly increases the activation Gibbs free energies by between 10 and $12 \mathrm{kcal}^{\mathrm{mol}} \mathrm{m}^{-1}$, which indicate that the most favorable reaction path corresponds to the formation of the product via TSout which are completely agreement with experimental results 4,5 . The structure of all TSout and TSins is given in Table 7.

Table 6. Energy $(\Delta \mathrm{E})$, Enthalpy $(\Delta \mathrm{H})$ and Gibbs free energy $(\Delta \mathrm{G})$, in $\mathrm{kcal} / \mathrm{mol}$, for the diepoxidation reaction paths between PaOUT, PyOUT, and m-CPBA.

\begin{tabular}{|c|c|c|c|}
\hline Compounds & $\Delta \mathbf{E}$ & $\Delta \mathbf{H}$ & $\Delta \mathbf{G}$ \\
\hline PaOUT (3) & - & - & - \\
\hline PyOUT (4) & - & - & - \\
\hline m-CPBA & - & - & - \\
\hline TSout (3) & 9.74 & 9.91 & 20.26 \\
\hline TSins (3) & 9.77 & 9.97 & 21.39 \\
\hline TSout (4) & 7.90 & 7.96 & 18.82 \\
\hline TSins (4) & 11.98 & 12.04 & 23.53 \\
\hline P' $\alpha O U T$ & -50.01 & -120.79 & -92.24 \\
\hline P' $\alpha I N S$ & -49.88 & -177.08 & -91.99 \\
\hline P'yOUT & -51.51 & -122.61 & -94.12 \\
\hline P'y INS & -49.19 & -120.16 & -91.48 \\
\hline
\end{tabular}

In diepoxidation reactions, the values of the GEDT are $0.35 \mathrm{e}$ at TSins $(3), 0.34 \mathrm{e}$ at TSout $(3), 0.33 \mathrm{e}$ at TSins (4), and $0.45 \mathrm{e}$ at TSout (4) (Table 7). The relatively high GEDT values found at the TSs, indicating the relatively polar character of these epoxidation reactions. All these results obtained theoretically are in agreement with those obtained experimentally ${ }^{4,5}$.

The asynchronicity of the formation of the bonds in the reaction studied can be measured by the difference between the lengths of the two $\sigma$ bonds forming in the reaction such that $\Delta d=\mid d(O 15-C 2)$ $\mathrm{d}(\mathrm{O} 15-\mathrm{C} 3) \mid$ (for $\alpha$-trans himachalene (1)). For the two reaction paths associated with the participation of the $\mathrm{C} 2=\mathrm{C} 3$ double bond of the (1), the asynchronicity at TSs is from 0.03 in TSout to 0.15 in TSins for monoepoxidation pathway. In diepoxidation pathway, the asynchronicity at TSs is from 0.01 in TSout to 0.02 in TSins (See Tables 8). In the case of $\gamma^{-}$trans himachalene (2), the asynchronicity at TSs is from 0.10 to TSout and from 0.20 to TSins in monoepoxidation reaction, and from 0.08 in TSout to 0.19 in TSins. From all these results, we can conclude that the TS is associated with the most favorable chemo-isomeric outside path, which is less asynchronous than that associated with the chemo-isomeric path inside (See Tables 9). 
Table 7. Optimized geometries of the stationary points involved in the diepoxidation reaction of the PaOUT (3), PyOUT (4) and m-CPBA. The lengths are given in angstroms, while the angles are given in degrees

TSins (3)

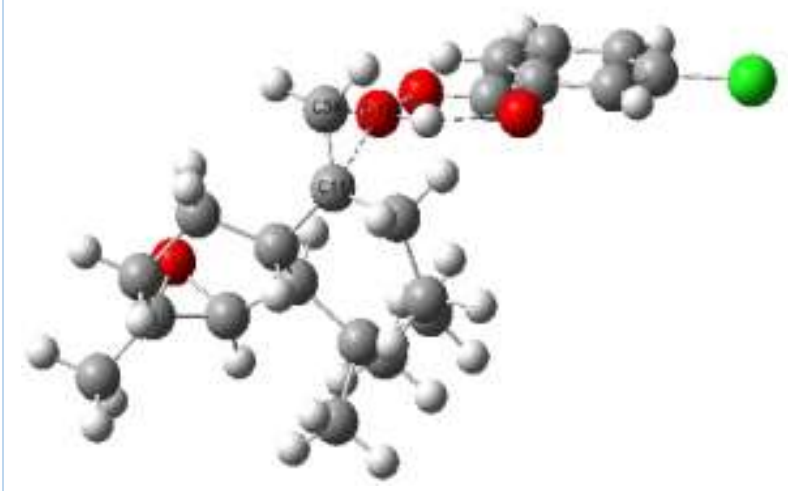

d(C11-015) $=2.559$

$\mathrm{A}(\mathrm{C} 11-015-\mathrm{C} 34)=32.24 \quad \mathrm{GEDT}=0.349$

TSins (4)

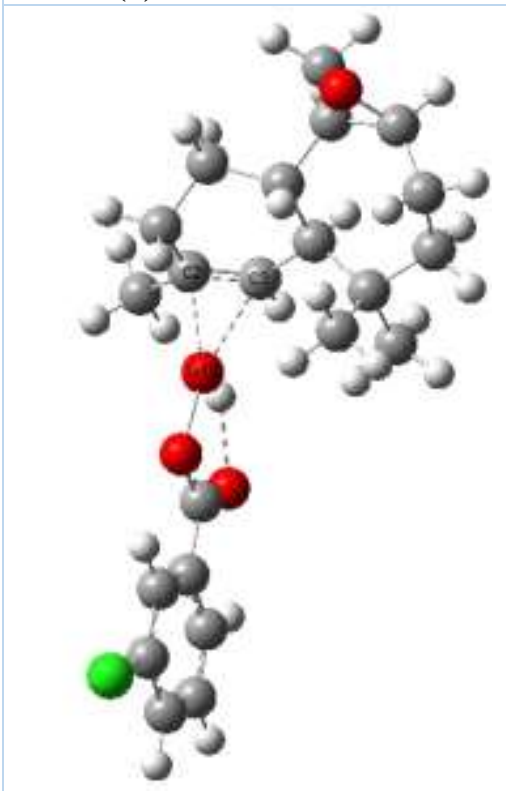

$\mathrm{d}(\mathrm{C2}-\mathrm{O15})=2.172 \quad \mathrm{~d}(\mathrm{C} 3-\mathrm{O15})=2.362$

$\mathrm{A}(\mathrm{C} 2-\mathrm{O15}-\mathrm{C3})=34.82 \quad \mathrm{GEDT}=\mathbf{0 . 3 3 8 \mathrm { e }}$
TSout (3)

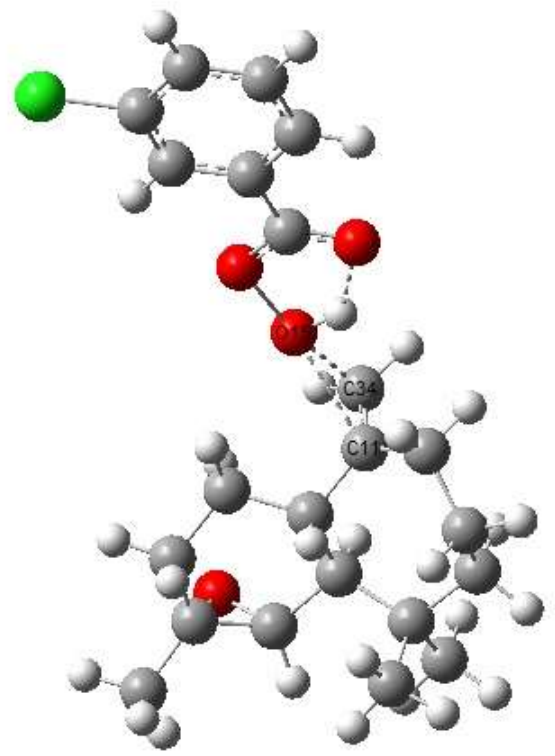

$\mathrm{d}(\mathrm{C} 11-\mathrm{O} 15)=2.436 \quad \mathrm{~d}(\mathrm{C} 34-\mathrm{O} 15)=2.109$

$\mathrm{A}(\mathrm{C} 11-\mathrm{O} 15-\mathrm{C} 34)=34.02 \quad$ GEDT $=\mathbf{0 . 3 5 0 e}$

TSout (4)

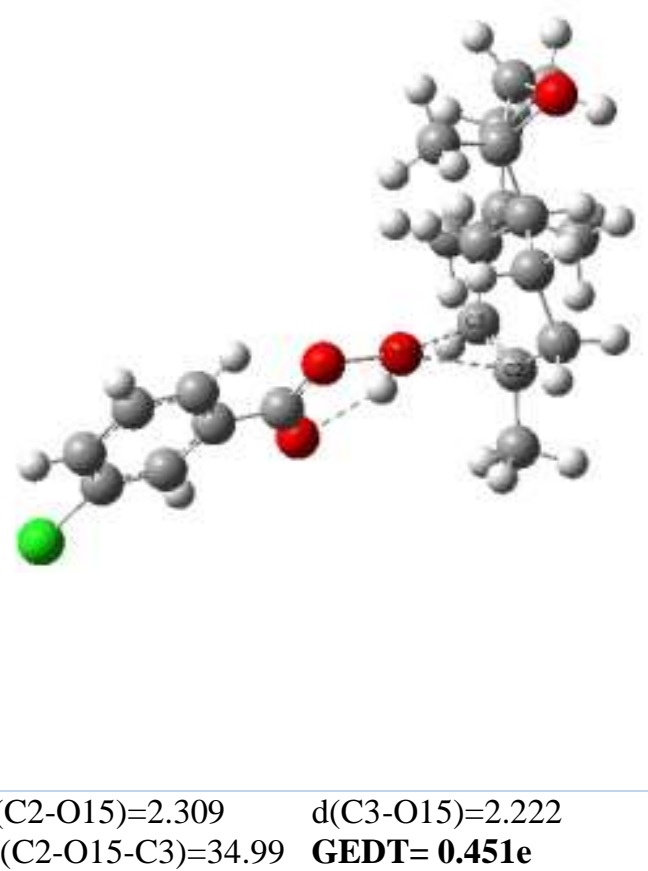

Table 8. Bond distances and bond differences (in Á) of the two newly formed bonds at the transition structures of Monoepoxidation and Diepoxidation of $\alpha$-trans himachalene (1).

\begin{tabular}{|l|l|l|l|l|l|l|}
\hline & \multicolumn{5}{|l|}{ Mode \#1 « TSout » } & \multicolumn{3}{l|}{ Mode \#2 « TSins » } \\
\hline Monoepoxidation & $d(\mathrm{O} 15-\mathrm{C} 2)$ & $d(\mathrm{O} 15-\mathrm{C} 3)$ & $\Delta d$ & $d(\mathrm{O} 15-\mathrm{C} 2)$ & $d(\mathrm{O} 15-\mathrm{C} 3)$ & $\Delta d$ \\
\cline { 2 - 8 } & 2.27 & 2.24 & 0.03 & 2.18 & 2.33 & 0.15 \\
\hline \multirow{2}{*}{ Diepoxidation } & $d(\mathrm{O} 15-\mathrm{C} 11)$ & $d(\mathrm{O} 15-\mathrm{C} 34)$ & $\Delta d$ & $d(\mathrm{O} 15-\mathrm{C} 11)$ & $d(\mathrm{O} 15-\mathrm{C} 34)$ & $\Delta d$ \\
\cline { 2 - 8 } & 1.44 & 1.43 & 0.01 & 1.45 & 1.43 & 0.02 \\
\hline
\end{tabular}


Table 9. Bond distances and bond differences (in $\AA$ ) of the two newly formed bonds at the transition structures of Monoepoxidation and Diepoxidation of $\mathrm{\gamma}$ - trans himachalene (2).

\begin{tabular}{|l|l|l|l|l|l|l|}
\hline & \multicolumn{3}{|l}{ Mode \#1 « TSout » } & \multicolumn{3}{l|}{ Mode \#2 « TSins » } \\
\hline Monoepoxidation & $d(\mathrm{O} 15-\mathrm{C} 11)$ & $d(\mathrm{O} 15-\mathrm{C} 13)$ & $\Delta d$ & $d(\mathrm{O} 15-\mathrm{C} 11)$ & $d(\mathrm{O} 15-\mathrm{C} 13)$ & $\Delta d$ \\
\cline { 2 - 8 } & 2.33 & 2.23 & 0.10 & 2.40 & 2.20 & 0.20 \\
\hline \multirow{2}{*}{ Diepoxidation } & $d(\mathrm{O} 15-\mathrm{C} 2)$ & $d(\mathrm{O} 15-\mathrm{C} 3)$ & $\Delta d$ & $d(\mathrm{O} 15-\mathrm{C} 2)$ & $d(\mathrm{O} 15-\mathrm{C} 3)$ & $\Delta d$ \\
& 2.30 & 2.22 & 0.08 & 2.17 & 2.36 & 0.19 \\
\hline
\end{tabular}

\section{Conclusion}

The chemo- and stereoselectivity of the reaction between $\alpha$ and $\gamma$ - trans himachalene and m-CPBA were studied within DFT method at B3LYP/ 6-311G $(\mathrm{d}, \mathrm{p})$ in dichloromethane as a solvent. The analysis of the global electrophilicity and nucleophilicity indices showed that the $\alpha$ - and $\gamma$-trans himachalene behave like a nucleophile, while the m-CPBA behaves like an electrophile. Regioselectivity found experimentally was confirmed by local electrophilicity indices and nucleophilicity $\omega_{\mathrm{k}}$ and $N_{k}$. The calculation of the activation energies shows for the two reactive paths associated with chemo and stereoselectivity approach modes of m-CPBA on $\alpha$ and $\gamma^{-}$trans himachalene have been analysed for both mono- and diepoxidation reactions. The Monoepoxidation of $\alpha$ and $\gamma$ - trans himachalene leads to the formation of two stereoisomers, on the most substituted double bond " $\mathrm{C}=\mathrm{C}$ " for $\alpha$ and $\mathrm{\gamma}^{-}$trans himachalene, respectively one of the two is the majority. Also, the diepoxidation reaction gives two more stereoisomers in the other carbon double bond for $\alpha$ and $\gamma$-trans himachalene, respectively.

\section{Acknowledgements}

This work was supported by Université Cadi Ayyad (UCAM).

\section{References}

1- F. Couic-Marinier, A. Lobstein, Les huiles essentielles gagnent du terrain à l'officine, Actualités pharmaceutiques, 2013, 52, 18-21.

2- A. Chekroun, A. Jarid, A. Benharref, A. Boutalib, Computational study of chemo-and stereoselectivity of $\alpha$-cis, $\alpha$-trans and $\alpha^{\prime}$-transhimachalene epoxidation by MCPBA, Journal of Molecular Structure: THEOCHEM, 2002, 588, 201-210.

3- M. Plattier, P. Teisseire, Essential oil of the Atlas cedar, Recherches, 1974, 19, 131-144.

4- A. Chiaroni, C. Riche, A. Benharref, H. El

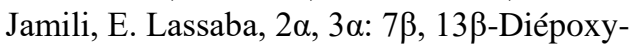
trans-himachalane, Acta Crystallographica Section C: Crystal Structure Communications, 1995, 51, 1171-1173.

5- A. Chiaroni, C. Riche, A. Benharref, H. El

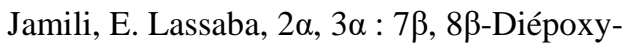

trans-himachalane, Acta Crystallographica Section C: Crystal Structure Communications, 1996, 52, 2502-2504.

6- M. A. Loriot, P. Beaune, La vitamine K époxyde réductase: du sang neuf dans les traitements anticoagulants oraux, La Revue de médecine interne, 2006, 27, 979-982.

7- M. R. Loizzo, A. M. Saab, G. A. Statti, F. Menichini, Composition and $\alpha$-amylase inhibitory effect of essential oils from Cedrus libani, Fitoterapia, 2007, 78, 323-326.

8- M. Aziz, F. Rouessac, Syntheses en serie racemique et en serie optiquement active d'une famille de derives oxygenes naturels de l'ombelliferone. Structure spatiale du (-) epoxy3'6'auraptene, Tetrahedron, 1988, 44, 101-110.

9- B. Frérot, M. Renou, C. Malosse, C. Descoins, Isolement et identification de composés à activité phéromonale chez les femelles de l'arctiide Tyria jacobaeae: détermination biologique de la configuration absolue du composé majoritaire, Entomologia experimentalis et applicata, 1988, 46, 281-289.

10- B. Satrani, M. Aberchane, A. Farah, A. Chaouch, M. Talbi, Composition chimique et activité antimicrobienne des huiles essentielles extraites par hydrodistillation fractionnée du bois de Cedrus atlantica Manetti, Acta botanica gallica, 2006, 153, 97-104.

11-G. Venkatesh, M. Govindaraju, P. Vennila, Experimental and theoretical spectral investigations of 5-chloro-ortho-methoxyaniline using FT-IR, FT-Raman and DFT analysis, Indian. J. Chem., 2016, 55A, 413-422.

12-M. J. Frisch, G. W. Trucks, H. B. Schlegel, G. E. Scuseria, M. A. Robb, J. R. Cheeseman, et al. Gaussian 03, Revision C.02, Gaussian, Inc, 2004.

13-R. Ghiasi, M. Z. Fashami, Tautomeric transformations and reactivity of isoindole and sila-indole: A computational study, J. Theor. Comput. Chem., 2014, 13, 1450041.

14-A. E. Reed, L. a. Curtiss, F. Weinhold, Intermolecular interactions from a natural bond orbital, donor-acceptor viewpoint, Chem. Rev., 1988, 88, 899-926.

15-H. Bernhard Schlegel, Optimization of equilibrium geometries and transition structures, J. Comput. Chem., 1982, 3, 214-218.

16- J. Tomasi, M. Persico, Molecular-interactions in solution - An overview of methods based on 
continuous distributions of the solvent, Chem. Rev., 1994, 94, 2027-2094.

17- E. Cances, B. Mennucci, J. Tomasi, A new integral equation formalism for the polarizable continuum model: Theoretical background and applications to isotropic and anisotropic dielectrics, J. Chem. Phys., 1997, 107, 3032-3041.

18- R. G. Parr, L. V. Szentpaly, S. Liu, Electrophilicity Index, J. Am. Chem. Soc., 1999, 121, 1922-1924.

19- R. G. Parr, R. G. Pearson, Absolute hardness: companion parameter to absolute electronegativity, J. Am. Chem. Soc., 1983, 105, 7512-7516.

20- R. G. Parr and W. Yang, Density Functional Theory of Atoms and Molecules, Oxford University Press, New York, 1989, pp. 333.

21- L. R. Domingo, E. Chamorro, P. Pérez, Understanding the Reactivity of Captodative Ethylenes in Polar Cycloaddition Reactions. A Theoretical Study, J. Org. Chem., 2008, 73, 4615-4624.

22- L. R. Domingo, P. Pérez, The nucleophilicity N index in organic chemistry, Org. Biomol. Chem., 2011, 9, 7168-7175.

23- W. Kohn, L. Sham, Self-Consistent Equations Including Exchange and Correlation Effects, $J$. Phys. Rev., 1965, 140, 1133-1138.

24-R. Huisgen, P. Pöchlauer, G. Mlostoń, K. Polborn, Reactions of di(tert-butyl) diazomethane with acceptor-substituted ethylenes, Helv. Chim. Acta., 2007, 90, 983-998.

25-S. A. Siadati, An example of a stepwise mechanism for the catalyst-free 1,3-dipolar cycloaddition between a nitrile oxide and an electron rich alkene, Tetrahedron Lett., 2015, 56, 4857-4863.

26-L.R. Domingo, J.A. Saez, J.A. Joule, L. Rhyman, $\mathrm{P}$, Ramasami, A DFT Study of the [3+2] versus $[4+2]$ Cycloaddition Reactions of 1,5,6-Trimethylpyrazinium-3-olate with Methyl Methacrylate, J. Org. Chem., 2013, 78, 1621-1629.

27-R. Jasiński, K. Mróz, A. Kącka, Experimental and Theoretical DFT Study on Synthesis of Sterically Crowded 2,3,3(4)5-Tetrasubstituted-4nitroisoxazolidines via 1,3-Dipolar Cycloaddition
Reactions Between Ketonitrones and Conjugated Nitroalkenes, J. Heterocycl. Chem., 2016, 53, 1424-1429.

28-X. Li, D. Wei, Z. Li, Theoretical study on DBUcatalyzed insertion of isatins into aryl difluoronitromethyl ketones: A case for predicting chemoselectivity using electrophilic parr function, ACS Omega, 2017, 2, 7029-7038.

29-E. Chamorro, P. Pérez, L.R. Domingo, On the nature of Parr functions to predict the most reactive sites along with organic polar reactions, Chem. Phys. Lett., 2013, 582, 141-143.

30-C. Morell, J.L. Gázquez, A. Vela, F. Guégan, H. Chermette, Revisiting electroaccepting and electrodonating powers: proposals for local electrophilicity and local nucleophilicity descriptors, Phys. Chem. Chem. Phys., 2014,16, 26832-26842.

31-R. Jasiński, M. Ziółkowska, O.M. Demchuk, A. Maziarka, Regio- and stereoselectivity of polar [2+3] cycloaddition reactions between $(Z)$ C-(3,4,5-trimethoxyphenyl)-N-methylnitrone and selected (E)-2-substituted nitroethenes, Cent. Eur. J. Chem., 2014, 12, 586-593.

32-A. K. Nacereddine, H. Layeb, F. Chafaa, A. Djerourou, L. R. Domingo, A DFT study of the role of the Lewis acid catalysts in the $[3+2]$ cycloaddition reaction of the electrophilic nitrone isomer of methyl glyoxylate oxime with nucleophilic cyclopentene, $R S C A d v ., 2015,5$, 64098-64105.

33- Z. Lakbaibi, A. Jaafar, H. Ben EL Ayouchia, M. Tabyaoui, A. Boussaoud, Reactivity and mechanism of nucleophilic addition reaction of amine with alkene: A systematic DFT study J. Mediterranean of Chemistry, 2019, 8(1), 25-29.

34- A. Benallou, Z. Lakbaibi, H. Garmes, H. El Alaoui EL Abdallaoui, The role of the polarity on the mechanism and selectivity in the $[3+2]$ cycloaddition reaction between $\mathrm{CF} 3$-ynone ylide and azide group: A quantum chemical investigation J. Fluorine. Chem, 2019, 219, 79-91.

35- L. R. Domingo, A new C-C bond formation model based on the quantum chemical topology of electron density, RSC Adv., 2014, 4, 32415-32428. 